\title{
THE EFFECT OF FEEDING NEUTRALIZER ON THE GROWTH OF BIFIDOBACTERIUM BIFIDUM
}

\author{
Guowei SHU*1, Shuai WANG*, He CHEN*, Man HU*, Tao QIN**, Qi MA** \\ *School of Food and biological Engineering, Shaanxi University of Science and \\ Technology, Xi' an, China \\ ** Enzyme Engineering Institute, Shaanxi Academy of Sciences, Xi'an, China
}

\begin{abstract}
In order to investigative the effect of different neutralizers and their feeding time on culture of Bifidobacterium bifidum BB01, and $\mathrm{pH}$, OD and viable count of B. bifidumBBO1 in the medium in different time were measured. The results indicated that the $\mathrm{NaOH}$ solution was the optimum neutralizer compared with the others, and feeding time of the neutralizer to $B$. bifidum BB01 was 13 hours after inoculation. Furthermore, the OD value and viable count reached maximum at $21 \mathrm{~h}(\mathrm{OD}$ value $=1.667)$ and $20 \mathrm{~h}$ (viable count: $(3.52 \pm 0.046) \times 10^{9}$ $\mathrm{CFU} / \mathrm{mL}$ ) after the $\mathrm{NaOH}$ solution was added to the medium, respectively. In addition, the maximum OD value implied that the logarithmic phase of B. bifidum BBO1 was delayed compared with the control and the viable count were $29.26 \%$ larger than the control group. The result of the study provides a method and an important basis for improving the viable counts of B. bifidum BBO1.
\end{abstract}

Keywords: probiotics, Bifidobacterium bifidum, neutralizers feeding, proliferation

\section{INTRODUCTION}

Bifidobacteria have raised attention due to their capability to adjust the immune response (Schultz, et al., 2004; Vissers et al., 2010) through their effects on human intestinal microflora. They are considered to have many beneficial effects on the host organism, including prevention and treatment of intestinal disorders, serum cholesterol reduction, stimulation of immune system, anti-carcinogenic activity, anti-mutagenic, produce antibiotics (Yusof, et al., 2000), improve the vitamin and protein metabolism, treating liver damage, relieve lactose intolerance and other functions (Arunachalam, 1999). Bifidobacteria are widely applied in fermented dairy products and probiotic adjuncts, such as yogurts and fermented milks (Ross et al., 2005), Sanchez et al., 2009).

\footnotetext{
${ }^{1}$ Corresponding author. Mailing address: Guowei Shu, 37\# School of Food and biological Engineering, Shaanxi University of Science \& Technology, Weiyang district, Xi'an city, Shaanxi Province, China. E-mail:shuguowei@gmail.com
} 
It is well known that the viable counts of the probiotic bacteria are the important factors on efficacy, transportation and storage, moreover, the growth of bacterial cultures vary depending on the growth medium and growth condition (Dietrich Knorr, 1998). On the one hand, it's well documented that the addition of sucrose (Costa et al., 2000; Palmfeldt et al., 2003), oligosaccharides (Falony et al., 2009), the plant cell wall polysaccharide arabinogalactan (Degnan and Macfarlane, 1995), fructose and inulin (Ozer and Akin, 2005) in media could increase the concentration of bifidobacteria in medium (Tao et al., 2007). Bifidobacteria were growing well on pure carrot juice without nutrient supplementation, the cell concentrations from $107 \mathrm{CFU} / \mathrm{mL}$ to $108 \mathrm{CFU} / \mathrm{mL}$ after $6 \mathrm{~h}$ of incubation (Szilard Kun et al., 2008). Janer et al. reported that caseinomacropeptide could be fed to complement milk in order to increase the number of bifidobacteria in probiotic fermented milks (Janer et al., 2004).

On the other hand, bfidobacteria fermentation accumulated acetic and lactic acids cause media acidification, which inhibit growth of this bacterium, because bifidobacteria are less acid-tolerant (Kailasapathy and Chin, 2000), (Gomes et al., 1999). The optimum $\mathrm{pH}$ value for growth of bifidobacteria is 6.5-7.0, and it inhibit growth when $\mathrm{pH}$ value below 5.0 or above 8.0 , furthermore, the acidic environment $(\mathrm{pH} \leq 5.5)$ is not suitable for cell growth (Du et al., 2009). Therefore, adding neutralizing agent to keep suitable acidity can make the viable count achieves a higher level.

In previous work, effects of ascorbic acid, cysteine hydrochloride, stachyose, xylooligosaccharide, galatooligosaccharide on growth of Bifidobacterium bifidum BB01 were studied (Shu et al., 2011),( Shu et al., 2013). The aims of the present work were to investigate three neutralizers $\left(\mathrm{NaOH}\right.$, ammonia, $\left.\mathrm{Na}_{2} \mathrm{CO}_{3}\right)$ and feeding time, which can encourage growth of Bifidobacterium bifidum BB01. The results will be helpful to provide a method for obtaining higher viable counts.

\section{MATERIALS AND METHODS}

Microorganism: The probiotic strains employed in the present study was Bifidobacterium bifidum BB01, which were obtained from School of Food and biological Engineering, Shaanxi University of Science and Technology.

Activation and cultivation method: Bifidobacterium bifidum BB01 freeze-dried powder were inoculated to MRS broth (Hope Bio-technology CO. LTD, Qingdao, China), and cultured $37{ }^{\circ} \mathrm{C}$ for $24 \mathrm{~h}$. Then $5 \%$ active culture was inoculated into the medium and incubated at $37{ }^{\circ} \mathrm{C}$ for $18 \mathrm{~h}$ until the viability of bacteria stays stable and the OD value reached a certain requirements.

Determination of $\mathbf{p H}$ : The $\mathrm{pH}$ of culture media was measured by a $\mathrm{pH}$ meter (pHS-3C Shanghai Precision Scientific Instrument Co., Ltd, Shanghai, China).

Determination of OD value: The optical density at $600 \mathrm{~nm}$ (OD600) was monitored through a spectrophotometer (SP-756PC, Shanghai Spectrum Co., Ltd., Shanghai, China). 
Determination of bacterial-growth curve: $5 \%$ active culture was inoculated into MRS broth. The growth media was incubated at $37{ }^{\circ} \mathrm{C}$ and sampled every two hours to measure the OD value, $\mathrm{pH}$ value and viable count, respectively. Draw the growth curve based on the experimental data.

Determination of viable count: Through a serial the gradient dilution on sterile saline solution, and used a syringe with $0.1 \mathrm{~mL}$ diluted bacterial suspension dropped into a count plate and then measured the viable counts.

The number of viable cells was determined by the spread plate technique using MRS agar (triplicate for each sample). Plates were anaerobically incubated at 37 ${ }^{\circ} \mathrm{C}$ for $48 \mathrm{~h}$, and the viable counts were represented by $\mathrm{CFU} / \mathrm{mL}$.

Cultivation of feeding test: Feeding time of the neutralizer and the influence of addition of neutralizers on $\mathrm{pH}$ and OD were studied. Preliminary determined the loading time of neutralizer, then determined the optimum neutralizing agent by studying the effect of different neutralizers on the viable counts.

\section{RESULTS AND DISCUSSIONS}

\section{The determination of feeding point time and optimal neutralizing agent}

The OD value and $\mathrm{pH}$ value of the medium were measured every one hour. The results were shown as Table 1 .

Table 1. The changes of OD and $\mathrm{pH}$ level by cultivation of Bifidobacterium bifidum

\begin{tabular}{|c|c|c|}
\hline culture time(h) & OD & $\mathrm{pH}$ \\
\hline 3 & 0.211 & 5.25 \\
\hline 4 & 0.345 & 5.17 \\
\hline 5 & 0.550 & 5.02 \\
\hline 6 & 0.808 & 4.85 \\
\hline 7 & 0.953 & 4.68 \\
\hline
\end{tabular}

The results showed that the $\mathrm{pH}$ of culture medium was gradually decreased and the OD value gradually increased in incubation time. The $\mathrm{pH}$ value closed to 5.0 at $5 \mathrm{~h}$ and in the sixth hour $\mathrm{pH}$ below 5.0. Due to the growth of bifidobacteria in $\mathrm{pH}$ below 5.0 become slowly (Du et al., 2009), the cell death occurred after 5 hours. Therefore, the point time at $5 \mathrm{~h}$ for adding neutralizing agent was chosen.

Furthermore, the selected neutralizing agent $(1 \mathrm{~mol} / \mathrm{L} \mathrm{NaOH}$ solution, $1 \mathrm{~mol} / \mathrm{L}$ $\mathrm{NaCO}_{3}$ solution and $2 \mathrm{~mol} / \mathrm{L}$ ammonia) were added to MRS broth after $5 \mathrm{~h}$, respectively. Then the $\mathrm{OD}$ value, viable counts and $\mathrm{pH}$ value of the medium were measured at $9 \mathrm{~h}, 12 \mathrm{~h}, 15 \mathrm{~h}, 18 \mathrm{~h}$, respectively (Figure 1). The $\mathrm{pH}$ value of each group decreased rapidly after $9 \mathrm{~h}$, it's showed that the neutralizer neutralized bacteria produced acid in the growth process. The viable counts were increase after feeding the neutralizer. Viable counts of the experimental group of adding $\mathrm{Na}_{2} \mathrm{CO}_{3}$ were increase gradually in $9-12 \mathrm{~h}$, and declined after $12 \mathrm{~h}$, that might be the $\mathrm{CO}_{2}$ produced by $\mathrm{Na}_{2} \mathrm{CO}_{3}$ could not be ruled out and dissolved in the medium decreased the acidity in the culture medium. 


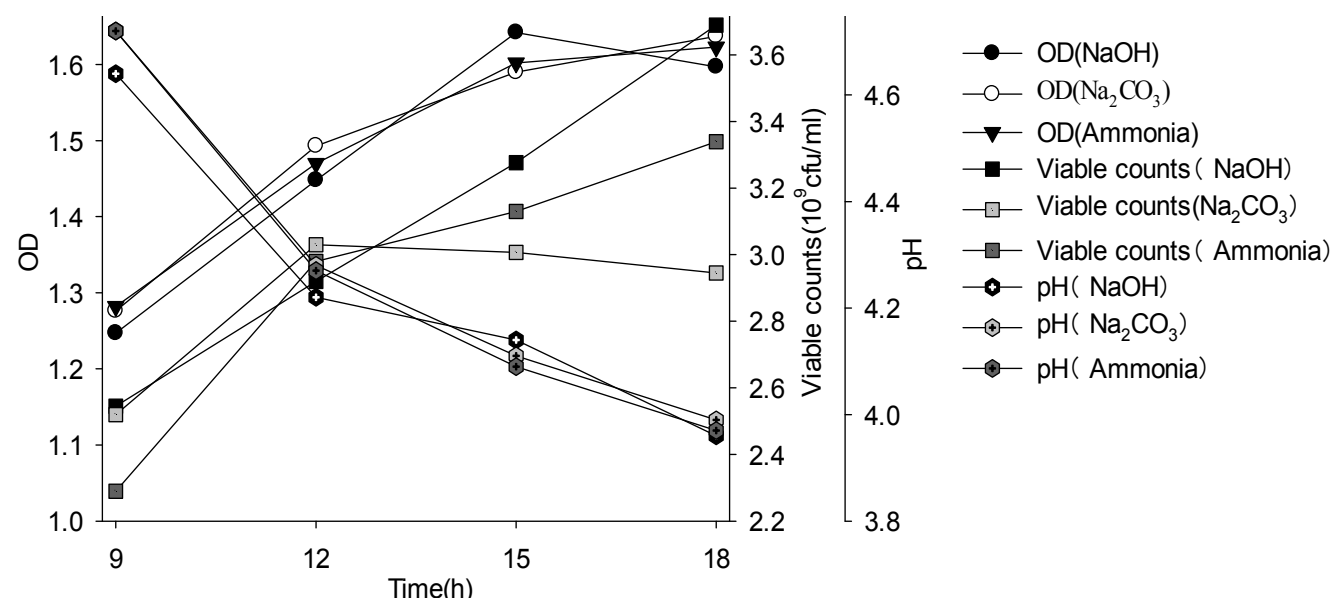

Figure 1. The effect of feeding alkali in medium on Bifidobacterium bifidum BB01. $\mathrm{OD}, \mathrm{pH}$ and viable counts.

Viable counts of the experimental group added ammonia were increase gradually in 9-12h, but increased more slowly after $12 \mathrm{~h}$. Viable counts of the experimental group adding $\mathrm{NaOH}$ were rapidly increased than the other two groups in 9-18h. This indicated that the neutralizer removed the influence of acid on the growth inhibition, especially $\mathrm{NaOH}$. The viable counts of the three experimental groups reached a maximum $3.69 \times 10^{9} \mathrm{CFU} / \mathrm{mL}, 3.03 \times 10^{9} \mathrm{CFU} / \mathrm{mL}, 3.34 \times 10^{9} \mathrm{CFU} / \mathrm{mL}$ at $18 \mathrm{~h}, 12 \mathrm{~h}, 18 \mathrm{~h}$, respectively. And the highest viable counts demonstrated that $\mathrm{NaOH}$ is the best neutralizing agent of Bifidobacterium bifidum BB01.

\section{Determination of feeding time}

The OD value, $\mathrm{pH}$ value and viable counts of the medium were measured during every $2 \mathrm{~h}$ to investigate the growth curve of Bifidobacterium bifidum BB01 (Figure 2 ). The $\mathrm{pH}$ value of the medium was reduced, which indicated that the proliferation of microorganisms produced a large amount of acidic substances. Bifidobacterium bifidum entered into logarithmic growth period at $4 \mathrm{~h}$ and reached the late logarithmic growth period at $13 \mathrm{~h}$. Number of viable cells began to decline after $18 \mathrm{~h}$. This showed that the proliferation and metabolism of Bifidobacteria and metabolic acid causes the reduction in $\mathrm{pH}$, which further inhibited the growth of bifidobacteria. Therefore, the late logarithmic growth phase (at 13h) was selected as the optimal feeding point time that added $\mathrm{NaOH}$ to neutralize the acid and prolong the logarithmic growth phase. 


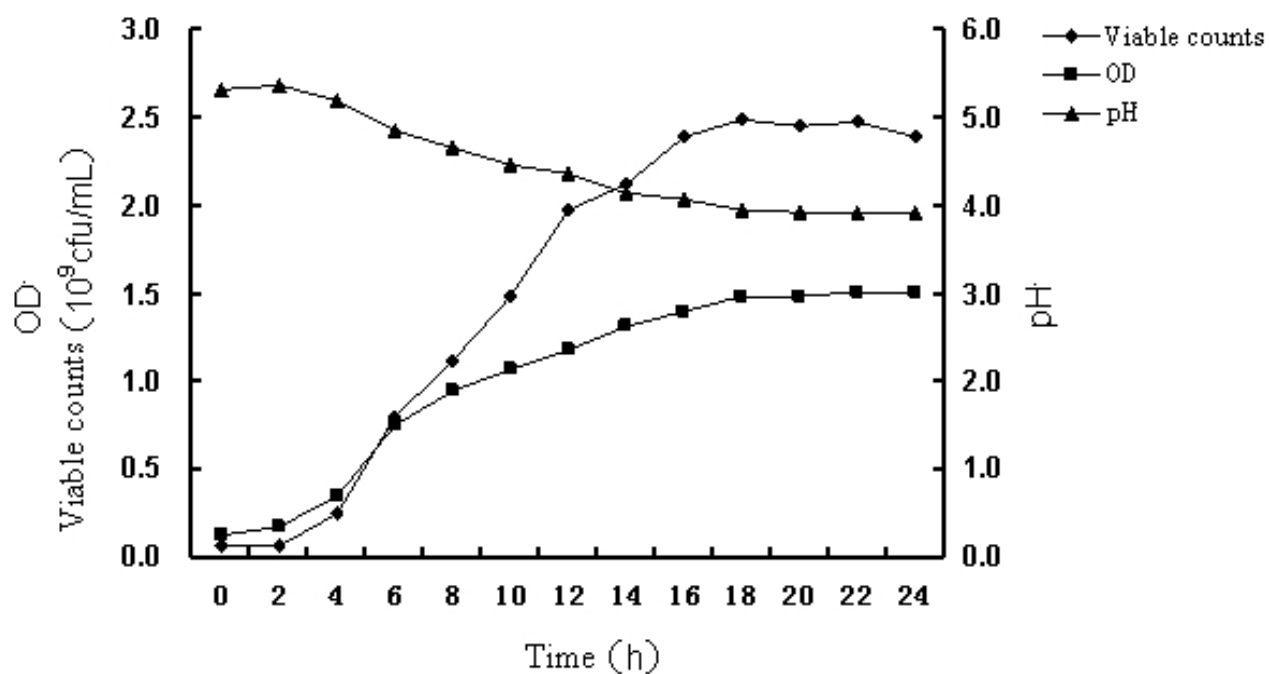

Figure 2. The effect of feeding alkali in medium on Bifidobacterium bifidum.OD, $\mathrm{pH}$ and viable counts.

\section{Effect of $\mathrm{NaOH}$ on the growth of microorganism}

Feeding (feeding $\mathrm{NaOH}$ at 13h) and control groups (without $\mathrm{NaOH}$ ) were compared by cultured under identical conditions. $\mathrm{NaOH}$ were added with a certain volume of $1 \mathrm{~mol} / \mathrm{L}$ to adjust the liquid $\mathrm{pH}$ value to 6.5 when Bifidobacterium bifidum $\mathrm{BB} 01$ were cultured to $13 \mathrm{~h}$, and then cultured to $24 \mathrm{~h}$. The $\mathrm{OD}$ value, $\mathrm{pH}$ value and viable count measured every $1 \mathrm{~h}$ after adding alkali, the results shown in Figure 3.

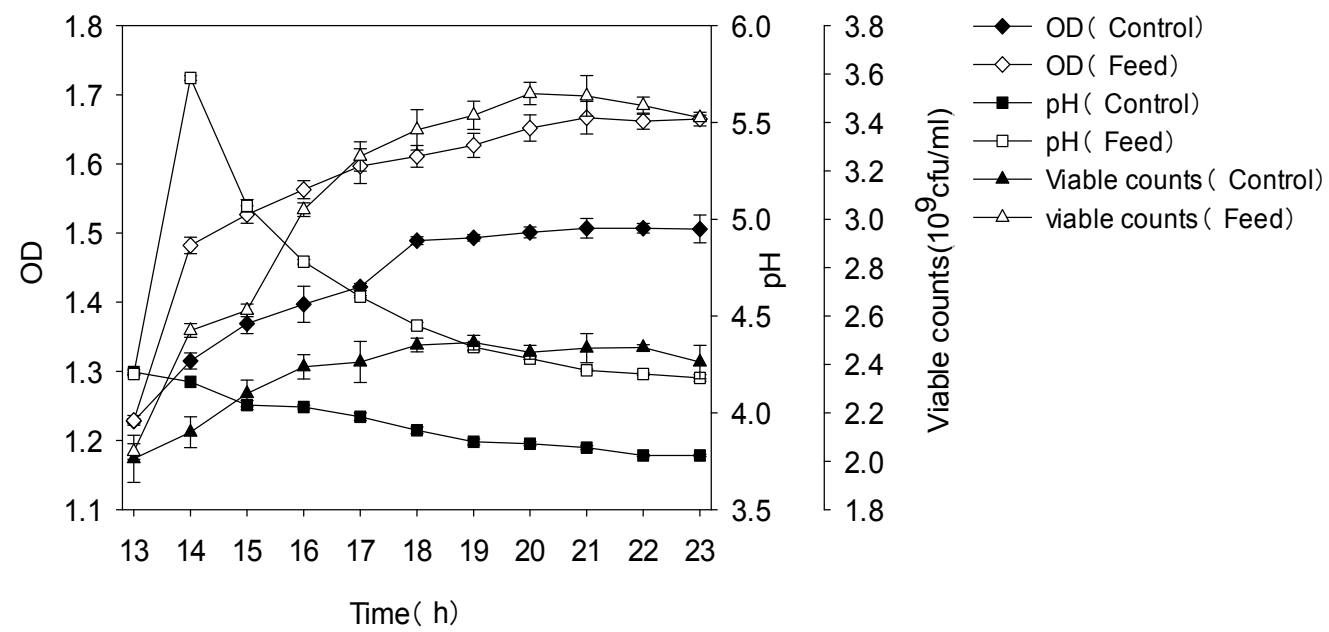

Figure 3. The result of verification test on feeding $\mathrm{NaOH}, \mathrm{OD}, \mathrm{pH}$ and viable counts.

The viable counts (control group) increased slowly after 13h, this was owing to accumulation of acid that inhibit the growth of Bifidobacterium bifidum BB01. On 
the contrary, after addition of $\mathrm{NaOH}$ at $13 \mathrm{~h}$, the $\mathrm{pH}$ value was higher and the viable counts increase again. In addition, viable counts of feeding group reached maximum at $20 \mathrm{~h}(3.52 \pm 0.046) \times 10^{9} \mathrm{CFU} / \mathrm{mL}$, a $29.26 \%$ increase than the control group $\left(18 \mathrm{~h}(2.49 \pm 0.03) \times 10^{9} \mathrm{CFU} / \mathrm{mL}\right)$. The OD value of feeding group reached 1.667 at $21 \mathrm{~h}$ can also suggest this. Compared with the control group, the time of cell logarithmic growth period was prolonged and reproductive capacity was enhanced. The $\mathrm{pH}$ value of the medium dropped to below 4.20 reached the late logarithmic growth after $21 \mathrm{~h}$, further proof that the logarithmic feeding could prolong the microbial growth and promoted cell growth. It was proved that the adding alkali extended the microbial logarithmic growth phase and bifidobactera enrichment effect was remarkable, viable counts in medium had a larger increase than before.

\section{CONCLUSIONS}

Addition of $\mathrm{NaOH}$ in MRS media could improve the buffer ability of MRS media and have the significant promotion on growth of Bifidobacterium bifidum. The effect of $\mathrm{NaOH}$ solution on bacterial growth was significantly, and the viable counts in medium reached the highest $(3.52 \pm 0.046) \times 10^{9} \mathrm{CFU} / \mathrm{mL}$ at $20 \mathrm{~h}$ when incubation, a $29.26 \%$ increase than the control group $\left(18 \mathrm{~h}(2.49 \pm 0.03) \times 10^{9}\right.$ $\mathrm{CFU} / \mathrm{mL}$ ) was reached. In conclusion, addition of $\mathrm{NaOH}$ played an important role in promoting growth of Bifidobacterium bifidum and provided reference for obtaining higher viable counts.

\section{ACKNOWLEDGEMENTS}

The project was partly supported by Education Department of Shaanxi Province (No. 2013JK0747), the Science and Technology Research Development plan project of Shaanxi Province (No. 2014K01-17-07), the science and technology plan project of Xi' an city [No.NC1317 (1)], China

\section{REFERENCES}

1. Arunachalam, K. D. (1999). Rioe of Bifidobcteria in nutrifion,medicine and technology. Nutr Res, 19,1559-1597.

2. Costa E, Usall J, Teixido N, et al. (2000). Effect of protective agents, rehydration media and initial cell concentration on viability of Pantoea agglomerans strain CPA-2 subjected to freeze drying. Journal of Applied Microbiology, 89(5), 793-800.

3. Degnan, B.A., Macfarlane, G.T. (1995). Arabinogalactan Utilization in Continuous Cultures of Bifidobacterium longum: Effect of Co-culture with Bacteroides thetaiotaomicron. Anaerobe, 1(2), 103-112.

4. Dietrich K., (1998). Technology aspects related to microorganisms in functional foods. Trends in Food Science \& Technology, 9, 295-306

5. Du, S. P., Yang, C. H., Shi, D. (2009). Breeding of Oxygen-resistant and 
Acid-resistant Bifidobacterium bifidum Strains. Modern Food Science and Technology, 25, 916-919.

6. Falony G., Calmeyn T., Leroy F., et al. (2009). Coculture fermentations of Bifidobacterium species and Bacteroides thetaiotaomicron reveal a mechanistic insight into the prebiotic effect of inulin-type fructans. Applied and Environmental Microbiology, 75(8), 2312-2319.

7. Gomes, A.M.P., Malcata, F.X. (1999). Bifidobacterium spp. and Lactobacillus acidophilus: Biological, biochemical, technological and therapeutical properties relevant for use as proboscis. Trend. Food Science \& Technology, 10, 139-157.

8. Janer, C., Pelaez, C., Requena, T. 2004. Caseinomacropeptide and whey protein concentrate enhance Bifidobacterium lactis growth in milk. Food Chemistry, 86(2), 263-267.

9. Kailasapathy, K., Chin, J. (2000). Survival and therapeutic potential of probiotic organisms with reference to Lactobacillus acidophilus and Bifidobacterium spp. Immunol. Cell. Biol., 78, 80-88.

10. Ozer, D., Akin, S., Ozer, B. (2005). Effect of inulin and lactulose on survival of lactobacillus acidophilus LA-5 and Bifidobacterium bifidum BB-02 in acidophilus-bifidus Yoghurt. Food Science and Technology International, $11(1), 19-24$.

11. Palmfeldt J, Radstrom P, Hahn-Hagerdal B. (2003). Optimisation of initial cell concentration enhances freeze-drying tolerance of Pseudomonas chlororaphis. Cryobiology, 47(1), 21-29.

12. Ross, R.P., Desmond, C., Fitzgerald, G.F., Stanton, C. (2005). Overcoming the technological hurdles in the development of probiotic foods. J.Appl. Microbiol., 98, 1410-1417.

13. Sanchez, B., de los Reyes-Gavilan, C. G., Margolles, A., \& Gueimonde, M. (2009). Probiotic fermented milks: Present and future. International Journal of Dairy Technology, 62, 472-483.

14. Schultz, M., Strauch, U.G., Linde, H.J., Watzl, S., Obermeier, F. (2004). Preventive effects of Escherichia coli strain Nissle 1917 on acute and chronic intestinal inflammation in two different murine models of colitis. Clin Diagn Lab Immunol, 11, 372-378.

15. Shu G., Ji Li-y., Chen H. (2011). Effects of stachyose, xylooligosaccharide and galatooligosaccharide on growth of Bifidobacterium bifidum. Food Science \& Technology, 36 (6), 6-9.

16. Shu G., Yang H., Qin T. and Chen H. (2013). Effect of Ascorbic Acid and Cysteine Hydrochloride on Growth of Bifidobacterium bifidum. Advance Journal of Food Science and Technology, 5(6), 678-681

17. Szilárd K., Judit M. Rezessy-Szabó, Quang D. Nguyen, et al. (2008). Changes of microbial population and some components in carrot juice during fermentation with selected Bifidobacterium strains. Process Biochemistry, 43(8), 816-821.

18. Tao H., Han R., Alvarez-Llamas G. et al. (2007). Differential analysis of Acta Universitatis Cibiniensis Series E: FOOD TECHNOLOGY 
protein expression of Bifidobacterium grown on different carbohydrates. Journal of Microbiological Methods, 69(2), 364-370.

19. Vissers, Y.M., Snel, J., Zuurendonk, P. F., Smit, B. A., Wichers, H.J. (2010). Differential effects of Lactobacillus acidophilus and Lactobacillus plantarum strains on cytokine induction in human peripheral blood mononuclear cells. FEMS Immunol Med Microbiol, 59, 60-70.

20. Yusof, R. M., Haqe, F., Ismail, M. (2000). Isolation of Bifidobacteria infantis and its antagonistic activity against ETEC 0157 and Salmonella typhimurium S-285 in weaning foods. Asia Pacific J Clin Nutr, 9, 130-135. 